\title{
Threat evaluation method of warships formation air defense based on $\operatorname{AR}(p)$-DITOPSIS
}

\author{
SUN Haiwen* and XIE Xiaofang \\ Coastal Defense College, Naval Aviation University, Yantai 264001, China
}

\begin{abstract}
For the target threat evaluation of warships formation air defense, the sample data are frequently insufficient and even incomplete. The existing evaluation methods rely too much on expertise and are difficult to carry out for the dynamic evaluation on time series. In order to solve these problems, a threat evaluation method based on the $\operatorname{AR}(p)$ (auto regressive (AR))-dynamic improved technique for order preference by similarity to ideal solution (DITOPSIS) method is proposed. The $\operatorname{AR}(p)$ model is adopted to predict the missing data on the time series. Then, the entropy weight method is applied to solve each index weight at the objective point. Kullback-Leibler divergence (KLD) is used to improve the traditional TOPSIS, and to carry out the target threat evaluation. The Poisson distribution is used to assign the weight value. Simulation results show that the improved $\operatorname{AR}(p)$-DITOPSIS threat evaluation method can synthetically take into account the target threat degree in time series and is more suitable for the threat evaluation under the condition of missing the target data than the traditional TOPSIS method.
\end{abstract}

Keywords: $\operatorname{AR}(p)$ model, Kullback-Leibler divergence (KLD), dynamic improved technique for order preference by similarity to ideal solution (DITOPSIS), time series, threat evaluation.

DOI: $10.21629 /$ JSEE.2019.02.09

\section{Introduction}

The threat evaluation [1] is an important part of making the warships formation air defense operation command decision, which is the foundation for commanders to set their determination and allocate firepower [2,3]. The modern high-tech local war shows that the five-dimensional war with the integration of land, sea, air, sky and electricity as the main air strike has become the main style of modern air attack, and it has implemented a large scale, multi level, multi batches and multi direction air raid on the target [4]. Thus, the threat evaluation process is challenging under normal situations due to limited time, stress and

Manuscript received June 17, 2017.

*Corresponding author.

This work was supported by the Postdoctoral Science Foundation of China (2013T60923). multi-tasking demands [5,6]. Human operators are responsible for performing the data analysis and proposing recommendations based on the data provided by the threat evaluation [7 -9]. It may propagate uncertainties and risks, making it imperative to reduce the cognitive load on operators while enhancing threat evaluation and decision making. Therefore, this requires an efficient and accurate threat evaluation method urgently.

At present, there are many methods for air target threat evaluation, such as multiple attribute decision making method [10-12], multi-criteria compromise solution sorting method [13,14], fuzzy theory method [15], rough set theory [16], Bayesian network [17-23] and so on. These methods are effective for specific problems, but they are still not applicable to the following situations. (i) Most of the evaluation methods rely too much on expert experience in the process of determining the weight of indices, which makes the accuracy of the evaluation results to depend on the expert experience credibility. The weight selection is not adaptive, and the flexibility is poor; thus this method is limited in an actual combat. (ii) Most of the methods can only give a fuzzy evaluation range and discrete qualitative results, which cause a low evaluation result resolution ratio. It cannot effectively use continuous quantitative indices. (iii) Most of the methods only use the current data instead of the comprehensive data in time series to carry out the threat evaluation. For the advanced hypersonic air target, because of the high velocity and superior maneuverability, the change of the threat index data on different time slices is obvious, which should be comprehensively carried out for the threat evaluation by combining data in time series. (iv) Most of the methods assume that the data is complete in the evaluation process. However, in the actual process, because of electromagnetic interference and large maneuverability, some data will often be missing and it is difficult to carry out the threat evaluation.

In order to solve these problems, we put forward an $\operatorname{AR}(p)$ (auto regressive (AR))-dynamic improved tech- 
nique for order preference by similarity to ideal solution (DITOPSIS) threat evaluation method. Aiming at the problem of quantitative data missing in threat indices, we use the self regressive $\operatorname{AR}(p)$ model to perform adaptive and fast data mending, which is combined with Bayesian information criterion (BIC) and the least square estimation. Combining with the complete data, avoiding the influence of subjective factors, the entropy weight method is used to make the objective empowerment of every threat index. Then, Kullback-Leibler divergence (KLD) is used to improve the traditional TOPSIS [24-27] method to sort the target threat, and improve the accuracy of the target threat ranking. Meanwhile, Poisson distribution is introduced to weight the time series, so that the data at the current time is associated with that at the previous time, which effectively reflects the dynamic threat characteristics of the target in time series. Finally, simulation experiments are carried out from three aspects: single time slice for multitarget threat evaluation, continuous multi-time slices for single target threat evaluation, and continuous multi-time slices for multi-target threat evaluation. We compare the proposed method with the projection method, the traditional TOPSIS and the improved TOPSIS, respectively, which verifies the feasibility, superiority and accuracy of the proposed method.

The remainder of this paper is organized as follows. Section 2 sets up an index system about the incoming target threat evaluation in warships formation air defense. Section 3 presents a description of data mending based on the $\operatorname{AR}(p)$ method. Section 4 describes time series weighting based on Poisson distribution. In Section 5, on the basis of Section 3, we describe the DITOPSIS target threat evaluation method combined with Section 4. In Section 6, we demonstrate the applicability and effciency of the proposed method. In Section 7, the conclusion is drawn.

\section{Setting up evaluation indices}

In the warships formation air defense operation, according to the number of different protected objects, the types of ship air defense can be divided into single warship air defense (the number of the protected ship is 1) and formation (or multi-ship) air defense (the number of the protected ship is more than 2). The threat evaluation of formation air defense is different from that of single ship air defense. The importance of the defended ship in the formation needs to be considered to evaluate the threat degree of the incoming target. The higher importance of the defended ship is, the higher threat degree of the incoming target is.

Threat evaluation indices should be selected according to the target data, which are detected and collected by various sensors in warships formation, and each index should be directly or indirectly related to the threat degree.
The threat evaluation indices of formation air defense are set up as shown in Fig. 1.

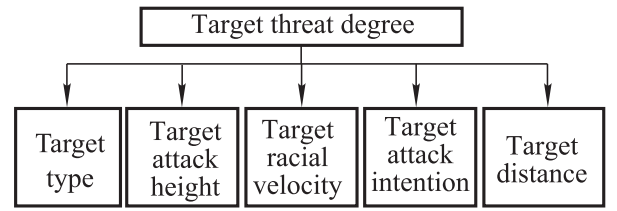

Fig. 1 Index system of formation incoming target threat evaluation

\subsection{Target type}

In the warships formation air defense operation, different target types have different threat degrees [28]. As the scope of formation air defense is increasing, the target types will be more complex. At present, the target types are distinguished into five categories: missile (class A), small aircraft (class B), helicopter (class C), large aircraft (class D), and air unknown target (class E), as shown in Table 1.

Table 1 Target type

\begin{tabular}{|c|c|c|}
\hline Type & Main target & Key features \\
\hline A & $\begin{array}{l}\text { All kinds of anti-ship mis- } \\
\text { siles, anti-radiation mis- } \\
\text { siles, guided bombs, attack } \\
\text { unmanned aerial vehicles } \\
\text { and so on }\end{array}$ & $\begin{array}{l}\text { The distance where they are found } \\
\text { is close, the target velocity is fast, } \\
\text { target reflection area is small, and } \\
\text { the target is a serious real threat. }\end{array}$ \\
\hline B & $\begin{array}{l}\text { Fighter aircraft, destroyer } \\
\text { aircraft, attack aircraft, } \\
\text { bombers }\end{array}$ & $\begin{array}{l}\text { Detection distance is relatively far, } \\
\text { the reflection area is relatively } \\
\text { large, and the target can constitute } \\
\text { a direct threat, which is a more seri- } \\
\text { ous real threat. }\end{array}$ \\
\hline $\mathrm{C}$ & Helicopter & $\begin{array}{l}\text { Slower; the target can constitute a } \\
\text { direct threat, which is a general re- } \\
\text { ality threat. }\end{array}$ \\
\hline $\mathrm{D}$ & $\begin{array}{l}\text { Early warning aircraft, } \\
\text { reconnaissance aircraft } \\
\text { (including reconnaissance } \\
\text { unmanned aerial vehicles), } \\
\text { tanker, anti-submarine air- } \\
\text { craft, jammers }\end{array}$ & $\begin{array}{l}\text { The distance where they are found } \\
\text { is far, the target does not constitute } \\
\text { a direct threat, but the enemy air } \\
\text { raid system is of great significance. }\end{array}$ \\
\hline $\mathrm{E}$ & Air UFOs & $\begin{array}{l}\text { They are serious threats based on } \\
\text { the principle of pessimism. }\end{array}$ \\
\hline
\end{tabular}

Class A and class E are assigned to 4, and class B, class $\mathrm{C}$, and class D are assigned to 3, 2, and 1, respectively. The index feature is that in a threat evaluation scenario, the larger the index value is, the more favorable the threat degree is.

\subsection{Target attack height}

The target height refers to the vertical distance from the target to the sea level [29]. In the process of data acquisition, the unit is $\mathrm{m}$. The index feature is that in a threat evaluation scenario, the lower the index value is, the more favorable the threat degree is. 


\subsection{Target radial velocity}

The target radial velocity refers to the velocity component on the line between the target and the defended ship [29]. In the process of data acquisition, the unit is Ma. The index feature is that in a threat evaluation scenario, the higher the index values are, the more favorable the threat degree is.

\subsection{Target attack intention}

The sketch map of the incoming target is shown in Fig. 2. When the anti-ship missile attacks my warships formation, if the target attempts to attack a certain ship, the target is considered to be a threat to the ship [29]. Normally, the enemy's attack intention can be judged by the target side angle. Suppose that at any time, a ship in my formation is located at the point $O$, the anti-ship missile $i$ is located at the point $T_{i}$, let $\theta_{j i}$ be the target side angle, $C_{i}$ be the heading, $D_{j i}$ be the distance from our ship, $\varphi_{j i}$ be the position related to our ship and $P_{j i}$ be the route shortcuts.

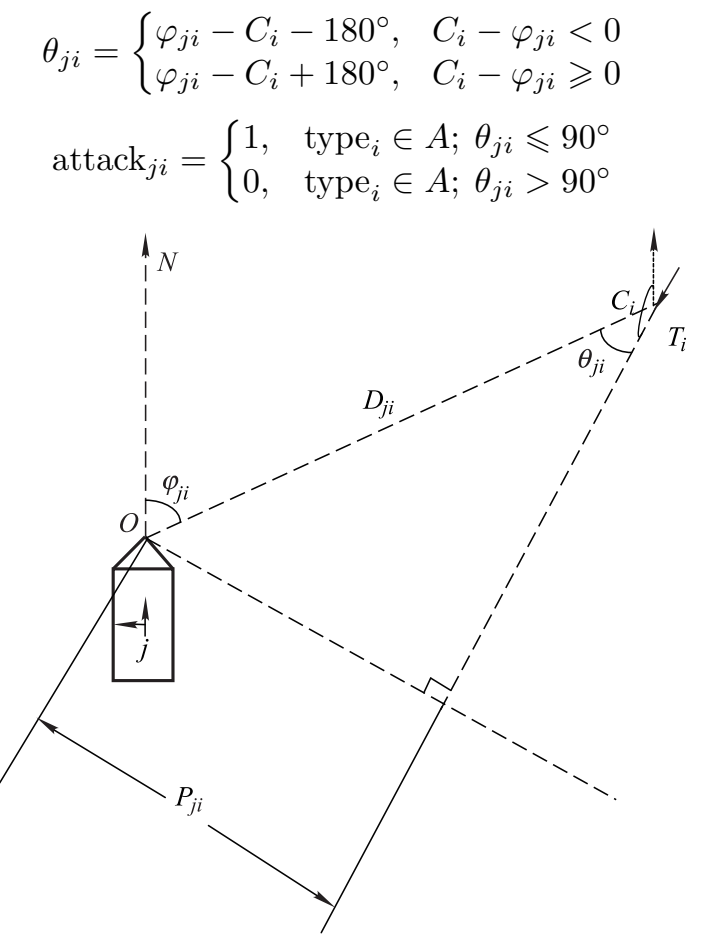

Fig. 2 Sketch map of incoming target

The attack intention is set as 1 , and no attack intention is set as 0 .

In the warships formation air defense operation, the attacked ship importance has an impact on assessing the incoming target threat degree. The higher the defended ship importance is, the higher the incoming target threat degree is. The ship importance on formation is determined according to the task nature currently carried out by the ship, the ability to accomplish the task and the ship value, themselves [30]. The types of ship importance are divided into "low", "middle" and "high". The ship importance index applies to the discrimination of the target attack intention.

The higher the ship importance is, the higher the threat attribute of the target attack intention is. The threat attribute types $\mu\left(T_{i}\right)$ of the attack intention are as follows:

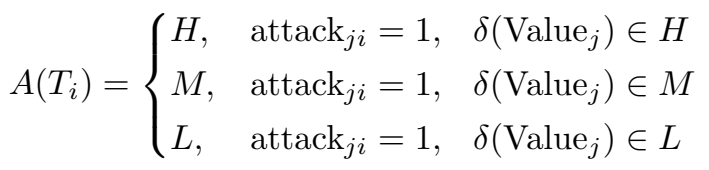

where, $H, M$ and $L$ express the importance for "high", "middle" and "low" respectively, and the values are 4, 3 and 2 , respectively.

In the actual process, when the incoming targets have attack intentions on multiple ships in the formation, the ship with the smallest target side angle is selected as the threatened object. If the target side angles of multiple ships are the same, the ship with high importance is selected according to the pessimistic principle. For a aircraft target, because the attack distance is far and the maneuverability range is large, when its target side angle exceeds $90^{\circ}$, the target has a certain threat intention, which is assigned to 1. The index feature is that in a threat evaluation scenario, the higher the index value is, the more favorable the threat degree is.

\subsection{Target distance}

The target distance refers to the relative distance between the target and the ship [29]. In the process of data acquisition, the unit is $\mathrm{km}$. The index feature is that in a threat evaluation scenario, the smaller the index value is, the more favorable the threat degree is.

\section{Data mending based on $\operatorname{AR}(p)$}

\subsection{Small sample data mending based on $\operatorname{AR}(p)$}

In the warships formation air defense operation, the quantitative observation data of the incoming targets are obtained by the constant interval sampling in time series, and there is a certain dependency relation between the data. The $\operatorname{AR}(p)$ model [31] can be used to predict the data.

The $\operatorname{AR}(p)$ model is defined as follows:

$$
X_{t}=a_{1} X_{t-1}+a_{2} X_{t-2}+\cdots+a_{p} X_{t-p}+u_{t}
$$

where $X_{t}$ is the observational data at the $t$ th moment, $\boldsymbol{a}=\left(a_{1}, a_{2}, \ldots, a_{p}\right)^{\prime}$ is an autoregressive parameter vector, and $u_{t}$ is the white noise with the order of $p$. Here, the $\operatorname{AR}(p)$ model is used to forecast data. Data in time series is required to be a stationary stochastic process; thus it is assumed that the sensor does not consider the influence of gross errors such as the human error operation and equipment fluctuation in the process of measuring data. 


\subsection{Determining the $\mathbf{A R}(p)$ order based on BIC criterion}

The BIC criterion is a criterion of model fitting goodness, and it is used to determine the $p$-value, which is defined as follows:

$$
\mathrm{BIC}(p)=\ln \widehat{\sigma}^{2}+p \ln N / N
$$

where $p$ is defined as the order of the model, $N$ expresses the number of the time series, $\widehat{\sigma}^{2}$ is defined as the variance of the fitting residual.

From the beginning that $p=1$, the autoregressive fitting of the observed data is obtained, and $\widehat{\sigma}^{2}$ is obtained. Then, the value of the order $p$ gradually increases and carries out fitting. With the increase of the order, the BIC value gradually decreases. At this point, $\widehat{\sigma}^{2}$ plays a decisive role. When increasing to a certain order, the BIC value reaches the minimum. Then, with the increase of the order $p$, the change amplitude of residuals variance is very small, but the order $p$ of the model plays a decisive role, that makes the BIC value to increase. At this time, let $p=p_{0}$, which is defined as the order of the best regression model.

\subsection{Parameter estimation based on least square method}

The parameter vector $\boldsymbol{a}=\left(a_{1}, a_{2}, \ldots, a_{p}\right)^{\prime}$ in the autoregressive model is estimated by using the least square method.

$$
\begin{gathered}
S(\boldsymbol{a})=\sum_{t=p+1}^{N} \sigma_{t}^{2}= \\
\sum_{t=p+1}^{N}\left(X_{t}-a_{1} X_{t-1}-a_{2} X_{t-2}-\cdots-a_{p} X_{t-p}\right)^{2}
\end{gathered}
$$

Let $\partial S(\boldsymbol{a}) / \partial \boldsymbol{a}=0$, the normal equations are given as follows:

$$
\left\{\begin{array}{c}
-2 \sum_{t=p+1}^{N}\left(X_{t}-a_{1} X_{t-1}-\cdots-a_{p} X_{t-p}\right) X_{t-1}=0 \\
-2 \sum_{t=p+1}^{N}\left(X_{t}-a_{1} X_{t-1}-\cdots-a_{p} X_{t-p}\right) X_{t-2}=0 \\
\vdots \\
-2 \sum_{t=p+1}^{N}\left(X_{t}-a_{1} X_{t-1}-\cdots-a_{p-1} X_{t-p+1}\right) X_{t-p}=0
\end{array}\right.
$$

From (4), it can be obtained as follows:

$$
\begin{gathered}
\left(\sum_{j=1}^{p} \sum_{t=p+1}^{N} X_{t-1} X_{t-j}\right) a_{1}+ \\
\left(\sum_{j=1}^{p} \sum_{t=p+1}^{N} X_{t-2} X_{t-j}\right) a_{2}+\cdots+
\end{gathered}
$$

$$
\left(\sum_{j=1}^{p} \sum_{t=p+1}^{N} X_{t-p} X_{t-j}\right) a_{p}=\sum_{j=1}^{p} \sum_{t=p+1}^{N} X_{t} X_{t-j} .
$$

Here, let

$$
\left\{\begin{array}{c}
\boldsymbol{A}=\left[\begin{array}{cccc}
X_{p} & X_{p-1} & \cdots & X_{1} \\
X_{p+1} & X_{p} & \cdots & X_{2} \\
\vdots & \vdots & \ddots & \vdots \\
X_{N-1} & X_{N-2} & \cdots & X_{N-p}
\end{array}\right] \\
\boldsymbol{\varphi}=\left[\begin{array}{c}
X_{p+1} \\
X_{p+2} \\
\vdots \\
X_{N}
\end{array}\right]
\end{array}\right.
$$

then the matrix equation can be obtained as follows:

$$
\left(\boldsymbol{A}^{\mathrm{T}} \cdot \boldsymbol{A}\right) \cdot \boldsymbol{a}=\boldsymbol{A}^{\mathrm{T}} \cdot \boldsymbol{\varphi} .
$$

Then the least square estimation $\widehat{\boldsymbol{a}}$ and $\widehat{\sigma}^{2}$ corresponding to $\boldsymbol{a}$ and $\sigma^{2}$ are obtained as follows:

$$
\widehat{\boldsymbol{a}}=\left[\begin{array}{c}
\widehat{a}_{1} \\
\widehat{a}_{2} \\
\vdots \\
\widehat{a}_{p}
\end{array}\right]=\left(\boldsymbol{A}^{\mathrm{T}} \cdot \boldsymbol{A}\right)^{-1} \boldsymbol{A}^{\mathrm{T}} \cdot \boldsymbol{\varphi}
$$

$\widehat{\sigma}^{2}=\frac{\sum_{t=p+1}^{N}\left(X_{t}-\widehat{a}_{1} X_{t-1}-\widehat{a}_{2} X_{t-2}-\cdots \widehat{a}_{p} X_{t-p}\right)^{2}}{N-p}$.

$\widehat{\sigma}^{2}$ is brought into (5) to obtain the value $p$, so that the suitable BIC model is obtained.

To sum up, in the process of the data repair based on the $\operatorname{AR}(p)$ model, the optimal $\operatorname{AR}(p)$ model parameters can be solved by the BIC criterion and the least square method. The model has the ability of self-adaptive repair, based on the numbers of different time series and different sample data values.

\section{Time series weighting based on Poisson distribution}

In the course of the target attack, the threat degree at the present moment is often affected by the target data in the previous time. Therefore, considering that the threat degree in time series will effectively reduce the number of the risk evaluation error. In order to evaluate the threat degree of the incoming target in the whole operation process, we need to weight different time slices in time series. It can be found that the closer it is to the present moment, the greater its contribution to the target threat evaluation is. The Poisson process refers to the increasing probability of "unexpected things" as time goes on. Therefore, the time series is 
weighted by the characteristics of the Poisson distribution [32]. The time series weight vector $\boldsymbol{\eta}=\left(\eta_{1}, \eta_{2}, \ldots, \eta_{n}\right)$ is calculated as follows:

$$
\eta_{k}=\frac{\frac{k !}{\varphi^{k}}}{\sum_{i=1}^{n} \frac{i !}{\varphi^{i}}}
$$

where $\eta_{k} \geqslant 0, \sum_{i=1}^{n} \eta_{k}=1,0<\varphi<2$.

\section{DITOPSIS threat evaluation method}

\subsection{Core thought}

The traditional TOPSIS [33] uses Euclidean distance to calculate the distance between the scheme and the positive (or negative) ideal solution. The specific calculation equation is as follows:

$$
\begin{aligned}
& S d_{i}^{+}=\sqrt{\sum_{j=1}^{n}\left(S_{j}^{+}-r_{i j}\right)^{2}}, \quad i=1,2, \ldots, m \\
& S d_{i}^{-}=\sqrt{\sum_{j=1}^{n}\left(S_{j}^{-}-r_{i j}\right)^{2}}, \quad i=1,2, \ldots, m
\end{aligned}
$$

where $r_{i j}$ denotes the weighted data, $S d_{i}^{+}$denotes the distance from the positive ideal solution, $S d_{i}^{-}$denotes the distance from the negative ideal solution.

$$
\begin{gathered}
S_{j}^{+}= \begin{cases}\max _{1 \leqslant i \leqslant m}\left\{r_{i j}\right\}, & j=1, \ldots n \\
\min _{1 \leqslant i \leqslant m}\left\{r_{i j}\right\}, & j=1 \ldots n\end{cases} \\
S_{j}^{-}= \begin{cases}\min _{1 \leqslant i \leqslant m}\left\{r_{i j}\right\}, & j=1, \ldots, n \\
\max _{1 \leqslant i \leqslant m}\left\{r_{i j}\right\}, & j=1, \ldots, n\end{cases}
\end{gathered}
$$

Since the Euclidean distance reflects the geometric distance between the two schemes, the points on the perpendicular between the two schemes cannot be distinguished and sorted. In order to solve this problem effectively, the KLD is introduced, which is asymmetrical and does not meet the trigonometric inequality. It is not the geometric distance of the two schemes, but it can effectively reflect the difference between the two schemes in practical applications. When the states of the two schemes are the same, the KLD value is zero; on the contrary, the greater the difference is, the greater the KLD value is. The concrete calculation is as follows:

$$
S d_{i}^{+}=\sum_{j=1}^{n}\left\{S_{j}^{+} \log _{10} \frac{S_{j}^{+}}{r_{i j}}+\left(1-S_{j}^{+}\right) \log _{10} \frac{1-S_{j}^{+}}{1-r_{i j}}\right\}
$$

$$
S d_{i}^{-}=\sum_{j=1}^{n}\left\{S_{j}^{-} \log _{10} \frac{S_{j}^{-}}{r_{i j}}+\left(1-S_{j}^{-}\right) \log _{10} \frac{1-S_{j}^{-}}{1-r_{i j}}\right\}
$$

As each variable in relative entropy obeys the discrete probability distribution, the index data should be normalized. In order to avoid the effect of the negative value in the index, (20) can be used.

$$
z_{i j}=\frac{x_{i j}}{\sqrt{\sum_{i=1}^{m}\left(x_{i j}\right)^{2}}}
$$

where $z_{i j}$ is the normalized value of $x_{i j}$.

\subsection{Evaluation steps}

Step 1 Establish the threat evaluation matrix:

$$
\boldsymbol{X}=\begin{gathered}
D_{1} \\
M_{1} \\
M_{2} \\
\vdots \\
M_{m}
\end{gathered}\left[\begin{array}{ccccc}
x_{11} & x_{12} & \cdots & x_{1 n} \\
x_{21} & x_{22} & \cdots & x_{2 n} \\
\vdots & \vdots & \ddots & \vdots \\
x_{m 1} & x_{m 2} & \cdots & x_{m n}
\end{array}\right]
$$

where $D_{n}$ denotes the threat evaluation index $n, M_{m}$ denotes the target $m, x_{m n}$ denotes the data of the threat evaluation index $n$ in the target $m$.

Step 2 Utilize the extreme standardization method to standardize the target threat evaluation matrix.

For the benefit criterion (the higher value is more favorable),

$$
v_{i j}=\frac{x_{i j}-\min \left(x_{j}\right)}{\max \left(x_{j}\right)-\min \left(x_{j}\right)} .
$$

For the cost criterion (the lower value is more favorable),

$$
v_{i j}=\frac{\max \left(x_{j}\right)-x_{i j}}{\max \left(x_{j}\right)-\min \left(x_{j}\right)} .
$$

where $x_{i j}$ denotes the data of index $j$ in the scheme $i, v_{i j}$ denotes the normalized value of $x_{i j} \cdot \max \left(x_{j}\right)$ and $\min \left(x_{j}\right)$ are the maximum and minimum of the index $j$, respectively.

Step 3 Calculate the feature weight of the evaluation object $i$ under the index $j$.

In view of the item $j$, the greater the difference between the values of $v_{i j}$ is, the greater the effect of this index on the evaluation object is. In other words, the more the useful information is provided to the evaluation object, the smaller the entropy is. The feature weight of the evaluation object $i$ under the index $j$ is calculated as follows:

$$
p_{i j}=\frac{v_{i j}}{\sum_{i=1}^{m} v_{i j}}
$$


where $0 \leqslant p_{i j} \leqslant 1$.

Step 4 Calculate the entropy value $e_{j}$ of the index $j$ :

$$
e_{j}=-\frac{\sum_{i=1}^{m} p_{i j} \ln \left(p_{i j}\right)}{\ln (m)} .
$$

When $p_{i j}=0$ or $p_{i j}=1, e_{j}=0$.

Step 5 Calculate the difference coefficient $d_{j}$ of the index $j$.

In view of the index $j$, the greater the difference between the values of $v_{i j}$ is, the smaller the value of $e_{j}$ is. When the values of index $j$ in each object are all equal, $e_{j}=1$. The difference coefficient

$$
d_{j}=1-e_{j} .
$$

$d_{j}$ is increasing gradually, which indicates that the index provides a large amount of information, and should be given a larger weight.

Step 6 Determine the entropy weight of each index:

$$
w_{j}=\frac{d_{j}}{\sum_{k=1}^{n} d_{k}}, \quad j=1,2, \ldots, n
$$

Step 7 Utilize the normalization method to deal with the threat evaluation matrix.

Step 8 Establish the weighted value:

$$
r_{i j}=w_{j} \cdot z_{i j} .
$$

Step 9 Use the KLD to calculate the positive and negative ideal solutions.

Step 10 Calculate the relative similarity degree between each scheme and the positive ideal solution:

$$
h_{i}=\frac{S d_{i}^{-}}{S d_{i}^{+}+S d_{i}^{-}} .
$$

Step 11 Establish the threat value matrix in time series:

$$
\boldsymbol{H}=\begin{array}{cccc}
T_{1} & T_{2} & \cdots & T_{n} \\
M_{1} \\
M_{2} \\
\vdots \\
M_{m}
\end{array}\left[\begin{array}{cccc}
h_{11} & h_{12} & \cdots & h_{1 n} \\
h_{21} & h_{22} & \cdots & h_{2 n} \\
\vdots & \vdots & \ddots & \vdots \\
h_{m 1} & h_{m 2} & \cdots & h_{m n}
\end{array}\right]
$$

where $h_{m n}$ denotes the threat value of the target $m$ on time slice $n$.

Step 12 Use the Poisson distribution to carry out the weighting of the time series, the weight vector $\boldsymbol{\eta}=$ $\left(\eta_{1}, \eta_{2}, \ldots, \eta_{n}\right) . \eta_{n}$ denotes the weight of time slice $n$.
Step 13 Integrate the target threat degree on each time slice to calculate the present target threat value $c_{i}^{n}$ :

$$
c_{i}^{n}=\boldsymbol{h}(i,:) \boldsymbol{\eta}^{\mathrm{T}}
$$

where $c_{i}^{n}$ denotes the target threat value of the target $i$ on the time slice $n . \boldsymbol{h}(i,:)=\left[h_{i 1}, h_{i 2}, \ldots, h_{i n}\right]$ denotes the threat value vector of the target $i$ on time slices $1-n$. $\boldsymbol{\eta}=\left(\eta_{1}, \eta_{2}, \ldots, \eta_{n}\right)$ denotes the weight vector of time slices $1-n$.

\section{Simulation experiment}

Assume that there are three ships in a certain ship formation to form a single column, including a destroyer $\left(K_{1}\right)$ and two frigates $\left(K_{2}\right.$ and $\left.K_{3}\right)$. Suppose that experts evaluate the importance of ships, $K_{1}$ is high, $K_{2}$ is medium and $K_{3}$ is low. In the past exercises, the sample data collected are recorded in a certain time period and used for simulation experiment.

\subsection{Data repair under missing samples}

Take the target height data as an example to repair the missing data, as shown in Table 2. The target data are sampled at the same interval at the beginning that the target is observed. In this process, electromagnetic interference or large maneuverability of the target lead to the loss of the target height data. As time goes on, the number of the missing data will increase, which will further affect the accuracy of the target threat ranking. When the sampling interval is small enough, any height change trend of the target will be reflected in the data sequence.

Table 2 Height values of incoming target in small sample data

\begin{tabular}{cc||cc}
\hline Time slice & Target height $h / \mathrm{m}$ & Time slice & Target height $h / \mathrm{m}$ \\
\cline { 2 - 4 }$t_{1}$ & 210.15 & $t_{6}$ & 170.55 \\
$t_{2}$ & 200.65 & $t_{7}$ & 153.78 \\
$t_{3}$ & 196.23 & $t_{8}$ & 131.22 \\
$t_{4}$ & 189.48 & $t_{9}$ & 112.36 \\
$t_{5}$ & 181.69 & $t_{10}$ & loss \\
\hline
\end{tabular}

The order of the optimal regression model is obtained as shown in Fig. 3.

It is known from Fig. 3 that the $p$ value is gradually increasing from the beginning of $p=1$. The BIC value is the minimum when $p=2$. Thus according to the BIC criterion, the model order $p$ is 2 . After determining the order of the model, the parameters estimation by using the least squares estimation is shown as follows:

$$
\widehat{\boldsymbol{a}}=[1.9232,-0.9345] .
$$




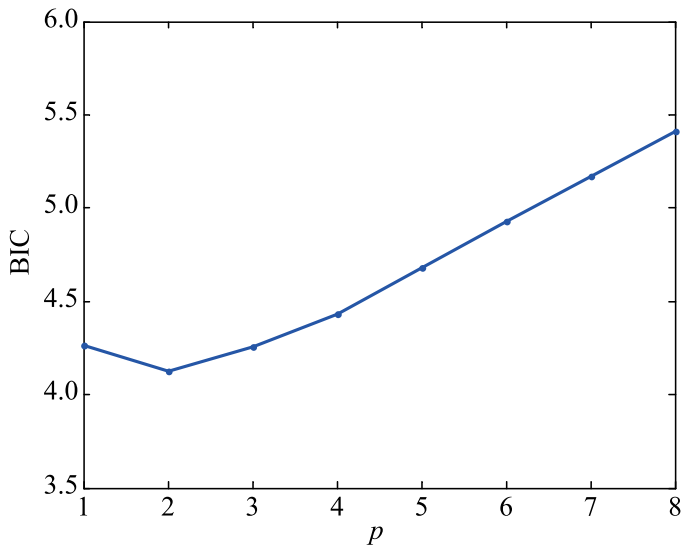

Fig. 3 Value of the model BIC under different orders

The prediction model is set up as follows:

$$
X_{t}=\left[X_{t-1}, X_{t-2}\right] \widehat{\boldsymbol{a}}^{\prime}
$$

As a result, the target prediction height at the time slice $t_{10}$ is $93.47 \mathrm{~m}$. It is known from Table 2, as time goes on, the downward trend of the target height is obviously increasing, and the threat degree is also increasing.

In the previous samples, all the sample data is assumed to be complete. But in the actual samples, the sample data is partially lost due to the electromagnetic interference or target maneuver, etc. In order to improve the accuracy of the ranking results, the $\operatorname{AR}(p)$ model can effectively predict the missing data and provide complete data for the subsequent target threat ranking.

\subsection{Single time slice multi-target threat evaluation and analysis}

Table 3 shows the complete multi-target data repaired on a certain time slice.

Table 3 Observation information of each target on a time slice

\begin{tabular}{cccccc}
\hline $\begin{array}{c}\text { Target } \\
\text { number }\end{array}$ & $\begin{array}{c}\text { Target } \\
\text { type }\end{array}$ & $\begin{array}{c}\text { Target } \\
\text { height/m }\end{array}$ & $\begin{array}{c}\text { Target radial } \\
\text { velocity/Ma }\end{array}$ & $\begin{array}{c}\text { Target attack } \\
\text { intention }\end{array}$ & $\begin{array}{c}\text { Target } \\
\text { distance/km }\end{array}$ \\
\hline 1 & 4 & 20.0 & 2.0 & 3 & 18.0 \\
2 & 1 & 8800.0 & 0.3 & 1 & 296.0 \\
3 & 4 & 25.0 & 1.1 & 3 & 45.0 \\
4 & 2 & 600.0 & 0.3 & 2 & 187.0 \\
5 & 3 & 120.0 & 1.3 & 3 & 180.0 \\
6 & 3 & 175.0 & 1.2 & 3 & 131.0 \\
\hline
\end{tabular}

According to the data in Table 3, the Projection method, traditional TOPSIS and improved TOPSIS (ITOPSIS) are used to get the threat degree of each target on present time slice to carry out ranking. The simulation results are shown in Figs. 4-6, respectively.

Fig. 4 shows the target threat degree calculated by using the Projection method. It is known that the target threat va- lues on this time slice are $0.5033,0.4967,0.3091$, $0.4279,0.3766,0.3326$, respectively, and the threat ranking result is target $1>\operatorname{target} 2>\operatorname{target} 4>\operatorname{target} 5>$ target $6>$ target 3 . According to the analysis, target 3 belongs to the missile class. Besides, the attack intention shows the threat degree is lower, while other indices show that the threat degree is higher. Thus theoretically the threat degree of target 3 should not be the minimum among the targets. However, in the actual ranking result, the threat degree of target 3 is the minimum, which indicates that the ranking results obtained by this method are not reasonable.

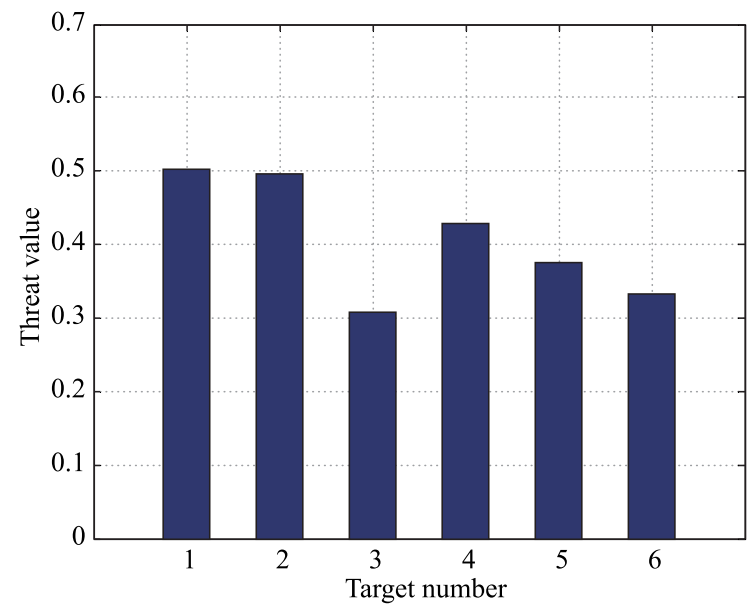

Fig. 4 Threat degree of each target by Projection method

Fig. 5 shows the target threat degree calculated by using the traditional TOPSIS. It is known that the target threat values on this time slice are $1.0000,0.0000,0.7121$, $0.2478,0.6874,0.6874$, respectively, and the threat ranking result is target $1>\operatorname{target} 3>\operatorname{target} 5=\operatorname{target} 6>$ target $4>$ target 2 . According to the analysis, there should be some differences between target 5 and target 6 , indicating that the ranking method cannot distinguish them.

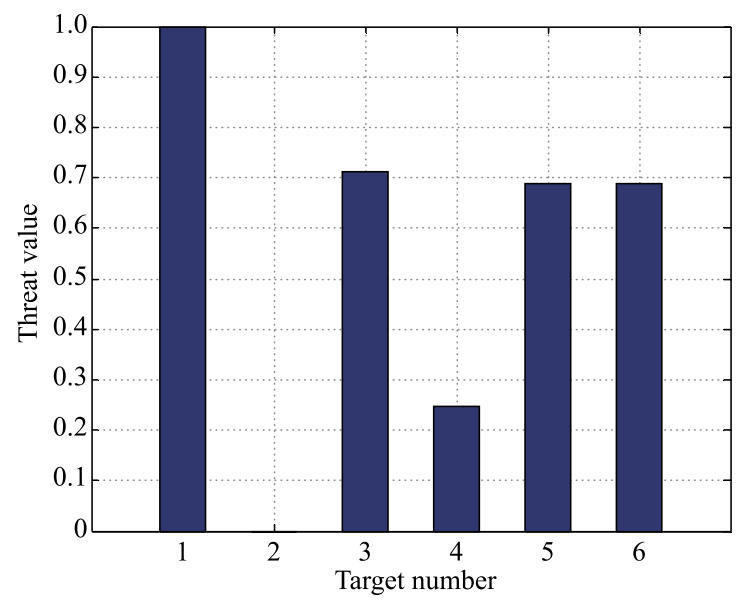

Fig. 5 Threat degree of each target by traditional TOPSIS

Fig. 6 shows the target threat degree calculated by using the ITOPSIS. It is known that the target threat values 
on this time slice are $1.0000,0.0000,0.9573,0.3910$, $0.8870,0.8962$, respectively, and the threat ranking result is target $1>\operatorname{target} 3>$ target $6>\operatorname{target} 5>\operatorname{target} 4>$ target 2 . According to the analysis, because the weight of the distance index is larger than that of the height index, the threat degree of target 6 is higher than that of target 5 . The ranking result is more reasonable.

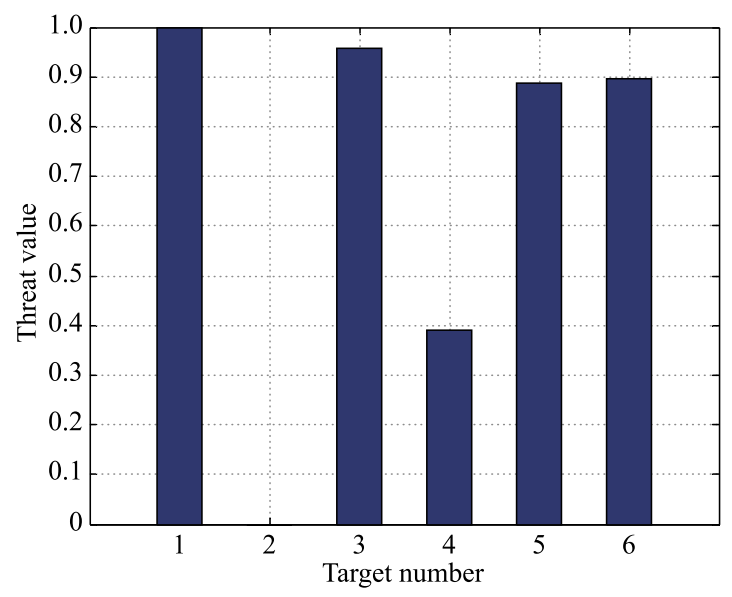

Fig. 6 Threat degree of each target by ITOPSIS

In conclusion, the resolution ability of the ITOPSIS method is much better than that of the projection method and the traditional TOPSIS method, which can effectively divide the threat degree of each target on the same time slice, and the ranking result is reasonable.

\subsection{Continuous multi-time slices single target threat evaluation and analysis}

Table 4 shows the observation data of a certain target on 10 consecutive time slices after repairing.

Table 4 Observation data of a certain target on continuous multitime slices

\begin{tabular}{cccccc}
\hline $\begin{array}{c}\text { Time } \\
\text { slice }\end{array}$ & $\begin{array}{c}\text { Target } \\
\text { type }\end{array}$ & $\begin{array}{c}\text { Target } \\
\text { height/m }\end{array}$ & $\begin{array}{c}\text { Target radial } \\
\text { velocity/Ma }\end{array}$ & $\begin{array}{c}\text { Target attack } \\
\text { intention }\end{array}$ & $\begin{array}{c}\text { Target } \\
\text { distance/km }\end{array}$ \\
\hline 1 & 1 & 10000 & 0.8 & 2 & 260 \\
2 & 3 & 8000 & 0.8 & 2 & 230 \\
3 & 2 & 6000 & 0.8 & 3 & 200 \\
4 & 1 & 4000 & 0.8 & 3 & 170 \\
5 & 3 & 2000 & 1 & 4 & 150 \\
6 & 3 & 800 & 1 & 4 & 140 \\
7 & 3 & 400 & 1 & 4 & 120 \\
8 & 3 & 1000 & 1.2 & 2 & 110 \\
9 & 3 & 2000 & 1.2 & 2 & 100 \\
10 & 3 & 3500 & 0.8 & 2 & 90 \\
\hline
\end{tabular}

According to the data in Table 4, the traditional TOPSIS, ITOPSIS and DITOPSIS are used to carry out threat ranking on the condition that the target type is ambiguous. The simulation results are shown in Fig. 7.

As can be seen from Fig. 7, the traditional TOPSIS cannot distinguish the target threat degree on the time slices 5 , 6 and 7. But the ITOPSIS and DITOPSIS in this paper can do that. Especially, the results obtained by the DITOPSIS method are more obvious.

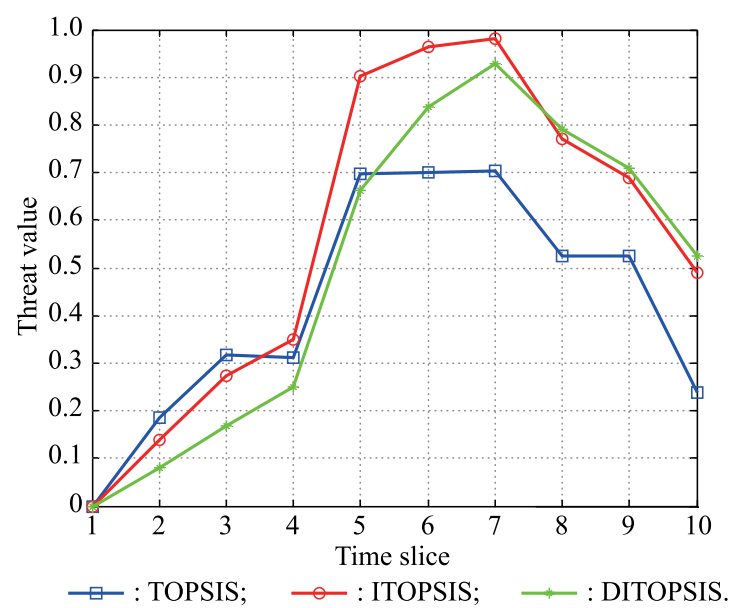

Fig. 7 Threat assessment of three methods on a single target continuous time slice

In the course of an actual combat, the target type cannot be identified effectively when the target distance is far, so the error is introduced. The three methods are used to carry out the threat ranking in two cases that the target type is clear and ambiguous. The simulation results are shown in Fig. 8.

As shown in Fig. 8, the curve fitting degree of the DITOPSIS method is better than that of the ITOPSIS and the traditional TOPSIS. In order to make a more intuitive and accurate comparison, the evaluation result is taken as a pseudo true value in the case that the target type is clear.

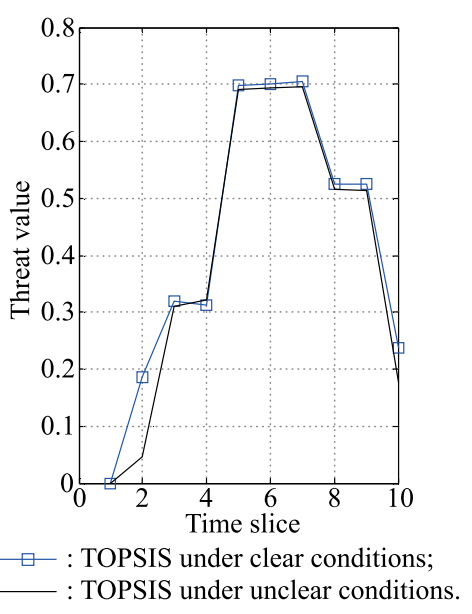

(a) TOPSIS 


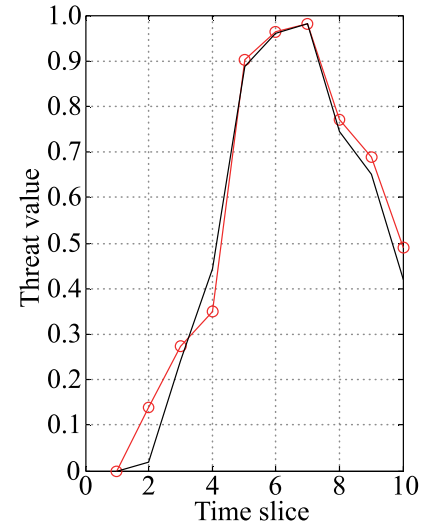

: ITOPSIS under clear conditions; : ITOPSIS under unclear conditions. (b) ITOPSIS

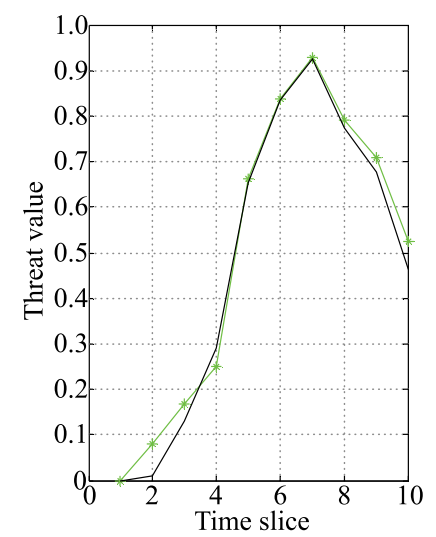

: DITOPSIS under clear conditions; : DITOPSIS under unclear conditions.

(c) DITOPSIS

Fig. 8 Assessment situation map of the three methods in two cases of clear and ambiguous target types

The residual standard deviation of the evaluation results in two cases are calculated. The residual standard deviation is also known as the standard deviation of error, which can reflect the reliability of the threat evaluation results when the target type is ambiguous. The equation is as follows:

$$
\frac{\delta=}{\sqrt{\frac{1}{n} \sum_{t=1}^{n}\left(\left|C_{\text {ambiguous }}^{t}-C_{\text {clear }}^{t}\right|-\frac{1}{n} \sum_{t=1}^{n}\left|C_{\text {ambiguous }}^{t}-C_{\text {clear }}^{t}\right|\right)^{2}}}
$$

where $\delta$ is the residual standard deviation, $C_{\text {clear }}^{t}$ denotes the threat evaluation value on the time slice $t$ when the target type is clear, $C_{\text {ambiguous }}^{t}$ denotes the threat evaluation value on the time slice $t$ when the target type is ambiguous, $n$ denotes the total number of time slices.

The simulation results are shown in Table 5.

As can be seen from Table 5, the ranking result of the residual standard deviation obtained by the three methods is: that of the DITOPSIS $<$ that of the ITOPSIS $<$ that of the traditional TOPSIS. Under the circumstances that the target type recognition error exists, this dynamic method can effectively reduce the impact of error on threat evaluation results, in order to improve the reliability of evaluation results.

Table 5 Residual standard deviation of the results of the three methods in two cases

\begin{tabular}{cc}
\hline Method & Residual standard deviation \\
\hline Traditional TOPSIS & 0.1637 \\
ITOPSIS & 0.0551 \\
DITOPSIS & 0.0326 \\
\hline
\end{tabular}

\subsection{Continuous multi-time slices multi-target threat evaluation and analysis}

(i) DITOPSIS and ITOPSIS simulation analysis

We take the time slice in Section 6.2 as the present time slice. It is assumed that the target data of the first two time slices are shown in Table 6.

Table 6 Threat index data of multiple targets on continuous time

\begin{tabular}{|c|c|c|c|c|c|c|}
\hline $\begin{array}{l}\text { Time } \\
\text { slice }\end{array}$ & $\begin{array}{c}\text { Target } \\
\text { number }\end{array}$ & $\begin{array}{c}\text { Targe } \\
\text { type }\end{array}$ & $\begin{array}{c}\text { Target } \\
\text { height } / \mathrm{m}\end{array}$ & $\begin{array}{l}\text { Target radial } \\
\text { velocity/Ma }\end{array}$ & $\begin{array}{c}\text { Target attack } \\
\text { intention }\end{array}$ & $\begin{array}{c}\text { Target } \\
\text { distance } / \mathrm{km}\end{array}$ \\
\hline \multirow{6}{*}{1} & 1 & 4 & 60 & 2.0 & 4 & 35 \\
\hline & 2 & 1 & 9000 & 0.3 & 2 & 300 \\
\hline & 3 & 4 & 80 & 1.2 & 4 & 56 \\
\hline & 4 & 2 & 1000 & 0.3 & 3 & 190 \\
\hline & 5 & 4 & 170 & 1.5 & 4 & 194 \\
\hline & 6 & 3 & 200 & 1.0 & 4 & 142 \\
\hline \multirow{6}{*}{2} & 1 & 4 & 40 & 2.0 & 4 & 27 \\
\hline & 2 & 1 & 8900 & 0.3 & 2 & 298 \\
\hline & 3 & 4 & 50 & 1.1 & 4 & 51 \\
\hline & 4 & 2 & 800 & 0.3 & 2 & 188 \\
\hline & 5 & 4 & 140 & 1.4 & 4 & 186 \\
\hline & 6 & 3 & 185 & 1.0 & 4 & 137 \\
\hline \multirow{6}{*}{3} & 1 & 4 & 20.0 & 2.0 & 4 & 18 \\
\hline & 2 & 1 & 8800 & 0.3 & 2 & 296 \\
\hline & 3 & 4 & 25 & 1.1 & 4 & 45 \\
\hline & 4 & 2 & 600 & 0.3 & 3 & 187 \\
\hline & 5 & 3 & 120 & 1.3 & 4 & 180 \\
\hline & 6 & 3 & 175 & 1.2 & 4 & 131 \\
\hline
\end{tabular}
slices

Based on the data of Table 6 , this dynamic method is used to simulate the threat evaluation results of time slice 3 , as shown in Fig. 9.

The target threat values on the time slice 3 are 0.9991 , $0,0.9550,0.3766,0.8926,0.8771$. The threat ranking result is target $1>\operatorname{target} 3>\operatorname{target} 5>\operatorname{target} 6>\operatorname{target} 4>$ target 2. Comparing the ranking results by the DITOPSIS and the ITOPSIS, the threat order between target 5 and target 6 is exchanged. From Table 6 , it can be seen that the target type of target 5 has changed on time slice 3 . The target type of target 5 on time slice 3 may be wrong through analyzing the data on the first two time slices. The evaluation results obtained by the DITOPSIS are integrated with the data on the first two time slices. The target type of target 5 in the present time is a larger probability as a missile 
type, and whose threat degree is greater than that of target 6 . The evaluation results are in line with the actual situation. In general, the DITOPSIS is better than the ITOPSIS, which can effectively reduce the impact of errors on the threat evaluation results, and improve the accuracy of the evaluation results.

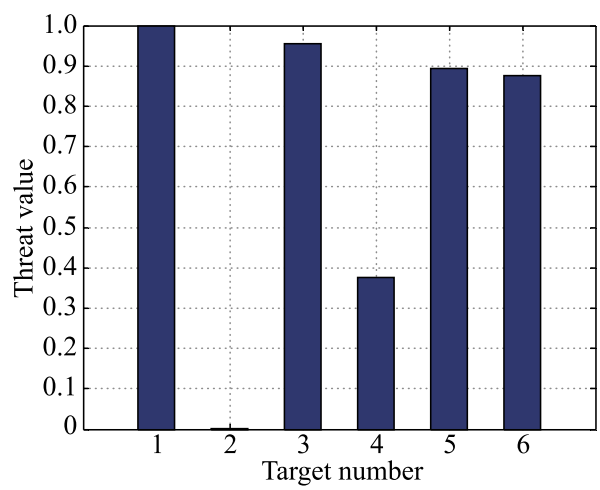

Fig. 9 Target threat value of time slice 3

(ii) Continuous multi-time slices multi-target threats evaluation and analysis

It is assumed that three targets are detected on a certain time slice, and the target types are missiles, helicopters, and fighter planes. The target data are sampled continuously on 20 time slices from time slice 0 . The threat degree of three targets on 20 continuous time slices is simulated by using this threat ranking method, as shown in Fig. 10.

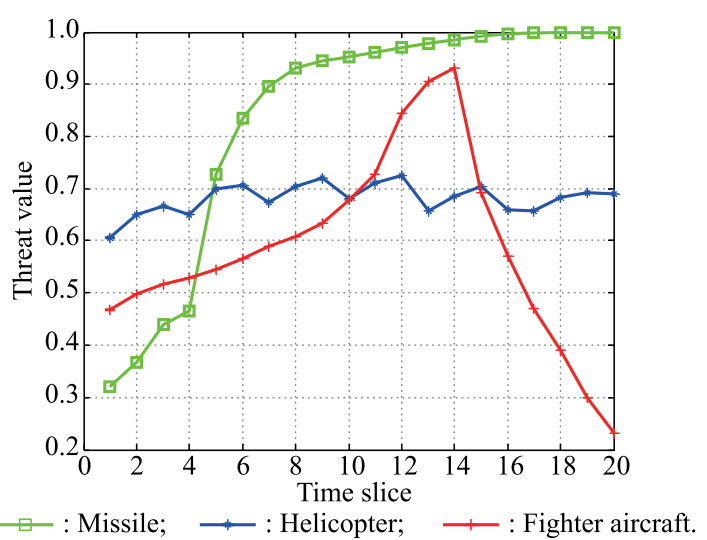

Fig. 10 Multi-targets threat situation map on continuous time slices

It can be seen from Fig. 10 that the velocity of the missile target is much faster, and its height decreases rapidly as the distance shortens, so the threat degree increases rapidly with time increasing, and is higher than that of the helicopter and fighter aircraft target. Since the helicopter target has a closer distance and a lower height, the threat degree is the maximum before time slice 4 . However, the velocity is slow, the maneuverability is poor, and the attack intention is ambiguous, so the threat degree varies slightly as time goes on. As time goes on, the velocity of fighter aircraft increases gradually, the height decreases and the distance shortens, so the target threat degree increases gradually. The target radial velocity is negative on time slice 14, which means that the target turns around and the threat degree drops rapidly. The simulation results are in line with the actual combat situation. The results are reliable, which can help decision-makers to make effective decisions based on the threat situation of multiple targets on continuous time slices.

\section{Conclusions}

The simulation experiment shows that the $\operatorname{AR}(p)$ model can effectively repair the missing data in the stationary random process. In the repair process, the optimal $\operatorname{AR}(p)$ model parameters are solved based on different time series numbers and different sample data values by the BIC and the least square method. The repair method has some adaptive repair ability.

The simulation results of the single time slice multitarget threat evaluation show that the traditional TOPSIS and the Projection method cannot give the threat ranking result, the ITOPSIS can effectively carry out the threat evaluation and ranking, and the ranking accuracy is higher than that of the traditional TOPSIS and the Projection method. On the basis of the ITOPSIS, the time weighting method of the Poisson distribution is introduced to carry out the dynamic threat ranking on the time series. The simulation results show that the proposed method can evaluate the threat degree in the present time slice by combining the data on time series, and effectively compensate for the threat evaluation error caused by uncertain data. The evaluation result is better than that of the ITOPSIS.

The simulation results of the continuous multi-time slice single target threat evaluation show that the threat evaluation result by the DITOPSIS is better than that by the ITOPSIS and the traditional TOPSIS. Under the circumstance that the target type is ambiguous, the proposed method has a certain fault-tolerant ability.

The simulation results of the continuous multi-time slices multi-target threat evaluation show that the threat evaluation results of this proposed method accord with the actual situation, and the reliability is high. The proposed method in this paper can help decision-makers to conduct command and decision-making based on the threat situation of multiple targets on continuous time slices.

\section{References}

[1] XU H, XING Q H, WANG W. Threat evaluation based on improved structure entropy and gray theory. Journal of Information Engineering University, 2016, 17(5): 620-625. (in Chinese)

[2] DAHLBOM A, HELLDIN T. Supporting threat evaluation through visual analytics. Proc. of the IEEE International Multi- 
disciplinary Conference on Cognitive Methods in Situation Awareness and Decision Support, 2013: 155-162.

[3] NASARUDDIN S H, LATIF L M A. Information system risk analysis using fuzzy techniques. Proc. of the International Symposium on Mathematical Sciences and Computing Research, 2013: MT-12.

[4] KARASAKAL O, OZDEMIREL N E, KANDILLER L. Antiship missle defense for a naval task group. Naval Research Logistics, 2011, 58: 305-322.

[5] AZIMIRAD E, HADDADNIA J. Target threat assessment using fuzzy sets theory. Advances in Intelligent Information, 2015, 1(2): $57-74$.

[6] NASEEM A, KHAN S A, MALIK A W. Real-time decision support system for resource optimization and management of threat evaluation and weapon assignment in air defense. Proc. of the IEEE International Conference on Industrial Engineering and Engineering Management, 2014: 565 -569.

[7] DENG Y, SU X, WANG D, et al. Target recognition based on fuzzy dempster data fusion method. Defense Science Journal, 2010, 60(5): $525-530$.

[8] ERLANDSSON T, NIKLASSON L. Automatic evaluation of air mission routes with respect to combat survival. Information Fusion, 2014, 20: $88-98$.

[9] RIVEIRO M, HELLDIN T, FALKMAN G, et al. Effects of visualizing uncertainty on decision-making in a target identification scenario. Computers and Graphics, 2014, 4: 84-98.

[10] PAN K, PAN X H, GUO X Q. Target threat judgment in surface antiaircraft based on MUDP. Computer and Digital Engineering, 2014, (5): 802-804, 821. (in Chinese)

[11] LI C F, ZHAO H, BA H X. Aerial target evaluation model based on multiple attribute decision making. Command Information System and Technology, 2011, 2(6): 55-58. (in Chinese)

[12] XU Y J, WANG Y G, MIU X D. Multi-attribute decision making method for air target threat evaluation based on intuitionistic fuzzy sets. Journal of Systems Engineering and Electronics, 2012, 23(6): $891-897$.

[13] ZHANG K, PIAO H Y, KONG W R. The improved VIKOR method based on dynamic parameters optimization in multitarget threat evaluation. Proc. of the 17th AIAA Aviation Technology, Integration, and Operations Conference, 2017: 1-8.

[14] YUAN Y, GUAN T, YAN X B. Based on hybrid VIKOR method decision making model for supplier selection. Control and Decision, 2014, 29: 551 - 558. (in Chinese)

[15] LEI Y J, WANG B S, WANG Y. Techniques for threat evaluation based on intuitionistic fuzzy reasoning. Journal of Electronics and Information, 2007, 29(12): 2805-2809. (in Chinese)

[16] FANG X Y, WANG H W, SUO Z Y. Radiator threat evaluating method based on rough set and information entropy. Journal of Beijing University of Aeronautics and Astronautics, 2016, 42(8): $1755-1760$

[17] OKELLO N, THOMS G. Threat evaluation using Bayesian networks. Proc. of the 6th International Conference on Information Fusion, 2003: $1102-1109$.

[18] KUMAR S, TRIPATHI B K. Modelling of threat evaluation for dynamic targets using Bayesian network approach. Procedia Technology, 2016, 24: 1268-1275.

[19] MENG G L, GONG G H. Threat evaluation of aerial targets based on hybrid Bayesian network. Systems Engineering and Electronic Technology, 2010, 32(11): 2398-2401. (in Chinese)

[20] WANG Y H, HAN Z P, CHEN S D. Analysis and modeling of threat evaluation system in air defense operations. Journal of Nanjing University of Aeronautics and Astronautics, 2014, 46(4): $558-565$.

[21] LIU Z, PENG J, HU Y N. A new kind dynamic Bayesian network and application to threat evaluation. Fire Control and
Command Control, 2014, 39(2): 16-20. (in Chinese)

[22] LIAO W H, QIANG J. Learning Bayesian network parameters under incomplete data with domain knowledge. Pattern Recognition, 2009, 42(11): 3046-3056.

[23] REN J, GAO X G, RU W. Paramenter learning of discrete dynamic Bayesian network with missing target data. Systems Engineering and Electronic Technology, 2011, 33(8): 1885 1890. (in Chinese)

[24] KUO T. A modified TOPSIS with a different ranking index. European Journal of Operational Research, 2017, 260: 152 160.

[25] SHAHER H, DANIELA F H. A bibliometric-based survey on AHP and TOPSIS techniques. Expert Systems With Applications, 2017, 78: 158 - 181. (in Chinese)

[26] ZHU J J, YI K K, JI D Q. Dynamic evaluation of urban credit environment in Jiangsu province based on entropy weight and TOPSIS. Journal of Nanjing University of Aeronautics and Astronautics, 2017, 19(2): 46-52.

[27] ZHU Q, XU S Q. Evaluation of influence of nodes in social network based on entropy-weight TOPSIS method. Journal of Nanjing University of Aeronautics and Astronautics, 2016, 18(1): $42-46$

[28] LIN H, WANG G B, WU C M, et al. Aerial target threat evaluation method for surface warship formation in air defense operation. Ship Electronic Engineering, 2016, 36(10): 16-18.

[29] ZHU B. Research on anti-air warfare command and decision for formation of ship-to-air missile. Dalian, China: Dalian Naval Academy of the PLA, 2013. (in Chinese)

[30] DAI J J, LI X M. Threat evaluation of air strike targets for warship formation networked air-defense operation. Systems Engineering and Electronics, 2013, 35(3): 532-538. (in Chinese)

[31] TANG X, YANG J J, FENG S, et al. AR( $p)$ dynamic catastrophe ranking method of target threat evaluation under the loss of data. Systems Engineering and Electronic Technology, 2017, 39(5): 1059 - 1064. (in Chinese)

[32] ZHANG K, WANG X, ZHANG C K. Evaluating and sequencing of air target threat based on IFE and dynamic intuitionistic fuzzy sets. Systems Engineering and Electronics, 2014, 36(4): 697 - 701. (in Chinese)

[33] ZHANG Y L, JI W P, LIU N N. Target threat evaluation based on entropy weight-topsis-grey correlation. Modern Defence Technology, 2016, 44(1): 72-78. (in Chinese)

\section{Biographies}

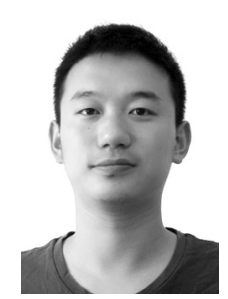

SUN Haiwen was born in 1990. He received his B.S. and M.S. degrees from Naval Aeronautical and Astronautical University (NAAU), Yantai, China in 2013 and 2016 respectively. He is currently pursuing his Ph.D. degree at the Coastal Defense College of Naval Aviation University. His main research interests are modeling and simulation of weapon systems.

E-mail: 842904820@qq.com

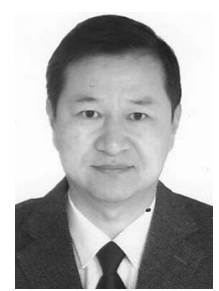

XIE Xiaofang was born in 1962. He is a professor and Ph.D. supervisor of the Coastal Defense College of Naval Aviation University. His current research interests are intelligent the design of weapon system and naval gun antimissile system.

E-mail: xiexf@yahoo.com.cn 